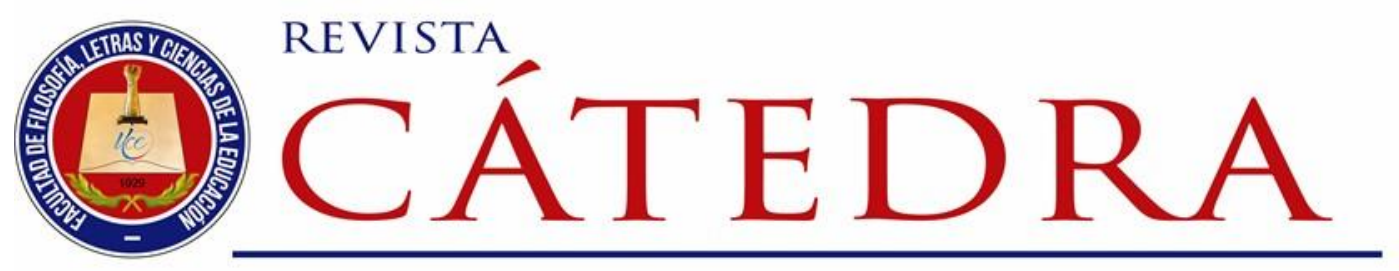

\title{
La propuesta metodológica como una alternativa para la integración de saberes
}

\author{
Methodological proposal as an alternative for the \\ integration of knowledge
}

\begin{abstract}
Floralba del Rocío Aguilar-Gordón
Universidad Politécnica Salesiana del Ecuador, Quito, Ecuador, Grupo de Investigación Filosofía de la Educación (GIFE), Quito, Ecuador

faguilar@ups.edu.es

https://orcid.org/0000-0002-9886-6878
\end{abstract}

(Recibido: 23/04/2019; Aceptado: 06/05/2019; Versión final recibida: 15/05/2019)

Cita del artículo: Aguilar-Gordón, F. (2019). La propuesta metodológica como una alternatica para la integración de saberes. Revista Cátedra, 2(2), 94-110.

\section{Resumen}

El presente artículo se propone reflexionar acerca de la propuesta metodológica como una alternativa de titulación, como una estrategia para la resolución de problemas y como una manera de integrar saberes que permitan la formación integral de los seres humanos; en tal sentido, este documento explica detalladamente cada una de las etapas de su constitución y analiza las características, contribuciones y estructura de la misma. Este trabajo pretende establecer un conjunto de pautas para la estructuración de una propuesta metodológica crítica, creativa e innovadora que responda a necesidades emergentes de la realidad educativa y de la sociedad en general. La propuesta metodológica se convierte en una estrategia pedagógica y didáctica que potencia diferentes habilidades, destrezas y competencias en el ser humano; es un referente para la formación profesional que valora los procesos investigativos, la percepción analítica, crítica y reflexiva de los diversos hechos, fenómenos y situaciones educativas-contextuales en las que se encuentran inmersos los sujetos. En el trabajo cotidiano del pedagogo en el salón de clase predomina la incertidumbre acerca de los procesos que se debería seguir para que el estudiante aplique los conocimientos adquiridos, de allí que el contenido expuesto en este manuscrito puede ser una oportunidad, un ejercicio y una acción a ejecutarse para que el estudiante pueda llevar a la práctica el corpus teórico adquirido. 
El documento se auxilia en el método fenomenológico-hermenéutico que permite un acercamiento directo al objeto de estudio y propicia la comprensión de los sujetos en su contexto.

\section{Palabras clave}

Educación, formación integral, integrador, propuesta metodológica, saberes.

\section{Abstract}

This article reflects on the methodological proposal as an alternative of qualification, as a strategy for solving problems and as a way of integrating knowledge that enables the integral formation of human beings; in this sense, this document explains the stages of its constitution and analyses its characteristics, contributions and structure. This paper aims to establish a set of guidelines for the structuring of a critical, creative and innovative methodological proposal that responds to the emerging needs of the educational reality and of the society in general. The methodological proposal becomes a pedagogical and didactic strategy that enhances different skills and competences in the human being; it is a reference for professional training that values the research processes, the analytical, critical and reflective perception of the facts, phenomena and educational-contextual situations in which the subjects are immersed. In the daily work of the professor predominates the uncertainty about the processes that should be followed in order for the student to apply the acquired knowledge; hence the content in this manuscript can be an opportunity and an action to be carried out so that the student can practice the acquired theoretical corpus. The manuscript uses the phenomenological-hermeneutic method that allows a direct approach to the object of study and promotes the understanding of the individuals in their context.

\section{Keywords}

Educación, formación integral, integrador, propuesta metodológica, saberes.

\section{Introducción}

El presente artículo se propone reflexionar acerca de la propuesta metodológica como una alternativa de titulación y como una manera de integrar saberes que permitan la formación integral de los seres humanos. Una propuesta metodológica es un tipo particular de manuscrito académico cuya misión principal es la comunicación de los resultados.

Así mismo, la propuesta metodológica constituye una de las alternativas para alcanzar la titulación en determinados programas de grado y de maestrías de profesionalización; son trabajos de titulación que tienen similar complejidad a los proyectos de investigación y de desarrollo; a los estudios comparados complejos; a los artículos científicos de alto nivel; al diseño de modelos complejos; a los productos artísticos; a los dispositivos de alta tecnología y a otros establecidos en el Reglamento de Régimen Académico (CES, 2017).

Las teorías científicas relacionadas con las propuestas metodológicas expresan que en esencia éstas se caracterizan por la instrumentación de un sistema de trabajo de integración interdisciplinar en el que intervienen una serie de elementos y aprendizajes con enfoque sistémico y procesual que se orientan a la puesta en práctica de aprendizajes, de habilidades, de competencias, de lo aprendido.

La propuesta metodológica se propone sistematizar un conjunto de saberes teóricos y prácticos que favorecen el alcance de aprendizajes significativos; se propician espacios en los que se evidencia el desarrollo de las competencias cognitivas, procedimentales y actitudinales desarrolladas por el estudiante a lo largo de un programa formativo

\section{Licencia Creative Commons Atribución 4.0 Internacional (CC BY 4.0)}

Revista Cátedra, 2 (2), pp. 94-110, mayo-agosto 2019. e-ISSN: 2631-2875

https://doi.org/10.29166/catedra.v2i2.1708 
determinado. De allí que, repensando de modo ontológico, el ser mismo de la propuesta metodológica se encuentra en la capacidad que desarrolla el sujeto para la reconstrucción de los saberes; en la habilidad para la renovación de las diversas formas de comprenderse y de entender al mundo y en las destrezas que despliega para la innovación críticaconstructiva- transformativa de la realidad en la que se encuentra.

La propuesta metodológica obliga al sujeto a realizar una integración de los saberes adquiridos con los nuevos conocimientos; su mente trabaja para concretar acciones en su cotidianidad, reestructura su mundo interno para modificar el mundo externo, lleva a la práctica el corpus teórico asimilado. Entre los problemas permanentes en torno a la estructuración de una propuesta metodológica se encuentran el qué se va a investigar; el cómo y en qué tiempo se va a hacer; el costo y los recursos que se van a utilizar. Estas interrogantes encontrarán una respuesta en el presente trabajo el cual pretende establecer un conjunto de pautas para la estructuración de una propuesta metodológica integradora, crítica, creativa e innovadora. Este trabajo se auxilia en el método fenomenológicohermenéutico que permite un acercamiento directo al objeto de estudio y propicia la comprensión de los sujetos en su contexto.

Este documento se encuentra estructurado por cinco partes: en la primera se aborda la caracterización de la propuesta metodológica; en la segunda se reflexiona acerca de las contribuciones que realizan este tipo de trabajos para el fortalecimiento y cumplimiento de los pilares de la educación; en la tercera se establecen algunas cuestiones preliminares referidas a la propuesta metodológica; en la cuarta se analizan las etapas del proceso de la propuesta metodológica y finalmente, en la quinta, se explica detalladamente la estructura de la propuesta metodológica. A continuación, se presenta el desarrollo de cada temática.

\section{Caracterización de la propuesta metodológica}

Entre las características de la propuesta metodológica se pueden mencionar las siguientes:

\subsection{Carácter integrador}

El carácter integrador de la propuesta metodológica hace realidad la formación integral del ser humano que se va autoconstruyendo convencido que el aprendizaje y el conocimiento se forja durante toda la vida. A medida que la sociedad se complejiza, "se vuelve prioritaria la revalorización de enfoques integradores, cualitativos y holísticos que tiendan a considerar que el conocimiento no se produce de manera lineal y que hace falta repensar el sentido, naturaleza, construcción y significado mismo del conocimiento" (Aguilar-Gordón, 2010, pág. 149). La nueva época exige modos de pensar colaborativos e integradores que superen los reduccionismos y la fragmentación de los saberes, requiere de nuevas maneras de interacción a partir del aparecimiento y del uso de las tecnologías digitales. Además, el carácter integrador de la propuesta metodológica efectiviza el hecho de que la educación es:

una realidad que pertenece al devenir espacio-temporal de todas las personas; es acción-creación, construcción y transformación de la vida en sociedad. La educación es un quehacer activo y de orden psico-físicosocial que permite la comprensión de nuevas realidades de acuerdo con el nivel de maduración interior de los sujetos (Aguilar-Gordón, 2019a, pág. 3). 


\subsection{Carácter interdisciplinar}

La propuesta metodológica es interdisciplinaria porque articula, dialoga, se relaciona y requiere la puesta en marcha de aspectos fundamentales de los diferentes saberes, disciplinas científicas o culturales que obligan a reconocer en los diferentes currículos la presencia de saberes filosóficos, epistemológicos, sociológicos y pedagógicos "como campos interdisciplinares rigurosos, con historicidad propia y como saberes que propician respuestas a la problematicidad humana de los últimos tiempos" (Aguilar-Gordón, 2019a, pág. 26).

\subsection{Carácter contextual}

La propuesta metodológica considera los rasgos característicos de los diversos ambientes educativos y la realidad tecnológica de los mismos, surge a partir de un diagnóstico y responde a un contexto educativo y social determinado en cuyo escenario pretende desarrollarse el conjunto de acciones tendientes a cumplir los objetivos propuestos. El carácter contextual de la propuesta metodológica propicia "un aprendizaje significativo sustentado en la experiencia, útil para la vida, ... con la incorporación de nuevos modelos y estrategias educativas..." (Aguilar-Gordón, 2019a, pág. 10) que promueven la necesidad de comprender y de responder a los requerimientos teóricos y praxológicos del propio entorno.

\subsection{Carácter motivador}

Este tipo de trabajos contribuye para que el sujeto tome conciencia del carácter ilimitado del conocimiento aspecto que le impulsa a dinamizarlo mediante la búsqueda de diferentes modalidades de estudios, en contextos reales y en entornos virtuales de aprendizaje.

\subsection{Carácter sistemático}

La propuesta metodológica cumple con procesos sistemáticos que procuran vincular y dar sentido a los contenidos programáticos abordados previamente en diversos módulos, cursos, y a las acciones del estudiante en el contexto educativo y social en el que se encuentra inmerso.

\subsection{Carácter innovador}

La propuesta metodológica y tecnológica se caracteriza por ser innovadora. En el ámbito pedagógico la innovación puede ser a nivel de generación de nuevos modelos educativos, pedagógicos y didácticos; la proposición de nuevas metodologías, técnicas y estrategias de enseñanza-aprendizaje; la incubación y construcción de nuevas herramientas e instrumentos de evaluación. La innovación puede ser, por ejemplo, "en el uso de estrategias poco convencionales, ... en el campo del cual podrán obtenerse valiosos resultados" (Marchetto, 2006, pág. 63) para todos los involucrados, para el docente en su rol de investigador, para el estudiante al obtener el aprendizaje por vías creativas, vivenciales y significativas y para el contexto al que se dirige.

La innovación en el ámbito tecnológico de la educación debería abarcar las cuatro fases sugeridas por Lara (1998): "la investigación, el desarrollo tecnológico, la aplicación y adopción, y el perfeccionamiento" (pág. 43) por cuanto en las propuestas la investigación contiene dos etapas fundamentales la investigación básica y la investigación aplicada. En referencia a la primera, la propuesta se orienta a la generación de conocimiento científico, sus centros de acción son las organizaciones, las instituciones educativas y en general el contexto socio-educativo. En relación a la segunda, la propuesta se direcciona hacia la búsqueda de alternativas de solución originales que coadyuven al mejoramiento de la situación actualmente existente. 


\subsection{Carácter cuestionador y resolutivo}

Se propone diagnosticar problemas y buscar alternativas de solución con miras a alcanzar la satisfacción de las diferentes necesidades educativas de los sujetos y de los contextos.

\subsection{Carácter organizativo}

Toda propuesta metodológica se desarrolla en base a planes, programas, proyectos y procesos organizados oportuna, coherente y sistemáticamente. La planificación se convierte en la columna vertebral de toda propuesta metodológica y tecnológica. Una adecuada planificación permite optimizar recursos, cumplir objetivos, satisfacer necesidades y expectativas individuales, sociales, académicas y pedagógicas fundamentales.

\subsection{Carácter formativo}

Las propuestas metodológicas y tecnológicas permiten cumplir con el objetivo central de la educación: "aprender a aprender" y con los cuatro pilares fundamentales de la misma: aprender a conocer, aprender a hacer, aprender a vivir juntos, aprender a ser, que fueron planteados por Delors (1996) en el informe a la UNESCO. En tal sentido, la educación deberá "propender al desarrollo de actividades meta-cognitivas útiles para la comprensión de la vida y del mundo; una educación que permita exponer lo que sentimos y lo que pensamos; una educación donde el estudiante hable más y escuche menos" (AguilarGordón, 2010, pág. 36).

\section{Contribuciones de la propuesta metodológica para el cumplimiento de los pilares de la educación}

- Aprender a ser. Las propuestas metodológicas y tecnológicas promueven la interacción directa o mediada por la tecnología con diversos sujetos, personalidades, contextos y realidades que lleva consigo el desarrollo de altos niveles de comprensión, de tolerancia, de adaptación a las circunstancias y el desarrollo de una elevada capacidad de autonomía, de responsabilidad y de respeto a la diversidad considerando los distintos estilos de aprendizaje, estilos de pensamiento, clases de memoria, capacidades físicas, aptitudes para comunicar, tipos de inteligencia, formas de razonamiento, etc.

- Aprender a conocer. La propuesta metodológica combina el corpus científico adquirido con la gama de conocimientos que emergen del entorno educativo social investigado. La articulación de la cultura general propia del maestrante con la sistematización de la teoría adquirida en el proceso formativo y las experiencias propias permiten la comprensión del problema, la profundización de la teoría que respalda a su propuesta y la generación de soluciones. Este tipo de trabajos le permiten aprender a conocer más para aprovechar todas las posibilidades que le ofrecen el fenómeno investigado, los sujetos y los contextos que intervienen.

- Aprender a hacer. La propuesta metodológica lleva consigo nuevos retos y desafíos para el maestrante puesto que no se reduce únicamente a la ejecución de un trabajo académico para alcanzar una cualificación profesional o una titulación, sino que va más allá, evidencia la puesta en práctica de todo cuanto sabe, descubre y proyecta con miras a responder a la diversidad de situaciones, experiencias y problemas educativos y sociales que le corresponde enfrentar.

- Aprender a vivir juntos. Las propuestas metodológicas promueven el desarrollo de la capacidad de interacción, de cooperación, de trabajo en equipo, de colaboración y de diálogo sostenido con los demás para encontrar las soluciones 
adecuadas para resolver conflictos y/o para solventar los diversos problemas educativos.

\section{Cuestiones preliminares de la propuesta metodológica}

Con la finalidad de propiciar el interés por la ciencia y su articulación con la praxis educativa y con las distintas situaciones de la actividad humana, la propuesta metodológica indaga acerca del qué, el cómo, el cuándo y el para qué del estudio de teorías y conceptos; establece una clara ruptura con lo memorístico y con el procedimiento de simple asociación para orientarse a la comprensión de nuevas problemáticas, contextos y sujetos. De allí que, las principales cuestiones preliminares de la propuesta metodológica sean los siguientes:

1. Identificación de las principales necesidades del contexto. Se convierte en la fase inicial de las propuestas metodológicas, revisa los antecedentes, las dificultades, describe los núcleos problemáticos presentes en una realidad determinada.

2. Problemática que se va a resolver. Es la base de toda propuesta, describe y explica el interés en los cambios y transformaciones reales que se quieren alcanzar.

3. Lluvia de ideas para la solución de la problemática. Generación y sistematización de ideas para la estructuración de una posible solución, deja abierto el camino para el trabajo de aplicación y de experimentación.

4. Métodos y técnicas para la aplicación y/o experimentación. Establecimiento de métodos, técnicas, procedimientos e instrumentos que permiten llevar a cabo la aplicación y experimentación de la teoría y de los conceptos involucrados en la situación problemática.

5. Conceptualización y fundamentación teórica de la alternativa de solución. Cada resultado exige profundización en la comprensión de los contenidos, una sólida sustentación teórica y su potencial aplicación.

6. Mecanismos de comunicación y socialización. Es necesario trabajar en las diversas representaciones y resultados de la exploración realizada, se busca diversos mecanismos como reportes, exposiciones, aplicaciones, informes, etc. que permitan la comunicación y socialización de la propuesta.

7. Revisión del proceso de construcción. Se realizan procesos de revisión, observación y corrección, se utilizan rúbricas, hojas de trabajo, guías que permitan una relectura y/o reelaboración de la propuesta.

8. Generación de pautas para la transferencia a otros escenarios. Se proponen pautas para aplicar y resolver problemas en otros contextos, se establecen nuevas ideas, preguntas y cuestionamientos dirigidos al análisis de otras realidades.

\section{Etapas del proceso de la propuesta metodológica}

La actividad investigadora de una propuesta metodológica y tecnológica se configura en función a tres grandes etapas:

1. Planificación de la investigación. Esta etapa se encuentra conformada por los siguientes pasos: la evaluación de las condiciones actuales y la definición del problema; la consulta de fuentes de información; la formulación de metas y objetivos; la formulación de hipótesis, proposición o idea a defender; identificación de variables o principales directrices; estructuración del plan de acción o del diseño mismo de la investigación que orientará a la propuesta; la asignación de recursos económicos, materiales, humanos y financieros que serán utilizados; los mecanismos de ejecución de la propuesta; y, finalmente, los mecanismos y estrategias de seguimiento y de control de la propuesta.

Licencia Creative Commons Atribución 4.0 Internacional (CC BY 4.0)

Revista Cátedra, 2 (2), pp. 94-110, mayo-agosto 2019. e-ISSN: 2631-2875

https://doi.org/10.29166/catedra.v2i2.1708 
2. Realización de la investigación. Esta etapa presupone los siguientes pasos: identificación del contexto, de los sujetos, de la población y de la muestra a utilizar, así como la identificación, organización y sistematización de los datos que se van a utilizar.

3. Obtención y comunicación de las conclusiones. Es la parte más breve e importante de los trabajos investigativos incluyen dos trabajos sucesivos: a) la elaboración de las conclusiones a las que se ha llegado luego de analizar e interpretar los datos obtenidos en el proceso investigativo; y b) la estructuración del informe de investigación que sirve como medio para comunicar los resultados, las dificultades encontradas, las limitaciones de la investigación, las proyecciones, así como las implicaciones en la práctica educativa.

\section{Estructura de la propuesta metodológica}

La propuesta metodológica se encuentra conformada por los siguientes elementos:

\subsection{Definición del tema}

El tema de la propuesta metodológica se caracteriza por ser breve, interesante, claro, preciso y atractivo para despertar el interés del lector. El tema deberá ser planteado de manera concisa, deberá ser informativo, no podrá exceder de un máximo de 85 caracteres con espacio incluido. El tema surge de las ideas; de las situaciones problemáticas; de una necesidad individual o social; de las experiencias teóricas o prácticas vividas por los expertos o por el propio investigador; de la observación de los contextos y de los sujetos; de los conocimientos previos del investigador; de las investigaciones realizadas; de las fuentes indagadas; etc.

\subsection{Resumen}

El resumen de una propuesta metodológica tendrá una extensión entre 220 palabras como mínimo y 250 palabras como máximo. El resumen se caracteriza por describir de forma clara y concisa los siguientes elementos: presentación breve del tema; formulación de objetivos y del problema; descripción del contexto de la problemática y de la metodología utilizada; presentación breve de los principales resultados y conclusiones. Debe estar escrito en forma impersonal, "el presente trabajo analiza..." (Aguilar-Gordón, 2019b, pág. 359).

\subsection{Problema}

El proceso de la estructuración de una propuesta metodológica y tecnológica avanzada se inicia en función a un área problemática de la que se extrae el problema de investigación; éste surge de un contexto teórico o práctico. Esta etapa a decir de Carrasco y Calderero (2009) puede sintetizarse en la pregunta “ide qué se trata?” (pág. 30).

La elección del problema exige claridad y precisión en el momento de su formulación, para elegir un problema idóneo, Pérez Juste et al. (1981) manifiestan que un problema puede surgir como:

a) fruto de la observación; b) resultado del contacto con profesores o personas expertas; c) consecuencia de lecturas de publicaciones, estudiando cómo se podrían aplicar a la solución de problemas las técnicas e ideas examinadas en ellas; d) conclusión de las charlas o discusiones entre especialistas; e) examen del apartado "discusiones" o 
"sugerencias" para futuras investigaciones que todo informe de investigación debe tener (pág. 96).

De manera que un problema de investigación bien formulado deberá reunir las siguientes condiciones: a) real (no construido o inventado por la mente del sujeto); b) realizable, esto es de acuerdo a Carrasco y Calderero (2009) "que pueda formularse una hipótesis como tentativa de solución y que sea posible comprobar dicha hipótesis" (pág. 31) o que pueda plantearse una proposición o idea a defender como tentativa de solución y de comprobación; y, c) original, a tal punto que no se encuentre resuelto o que la resolución haya sido parcial.

Es preciso que el problema sea formulado con precisión en una o varias preguntas concretas con la finalidad de que se constituya en una guía adecuada para la formulación de la hipótesis, la proposición o idea a defender.

\subsection{Objetivos}

Los objetivos de una propuesta metodológica y tecnológica son un conjunto de tareas fundamentales que se cumplen en la construcción de todo tipo de conocimiento científico. Los objetivos son aquellos fines, propósitos o metas específicas que se pretenden alcanzar "para poder responder a una pregunta de investigación y que orientan al desarrollo de la investigación" (Briones, 2013, pág. 8).

La propuesta metodológica debe plantear un objetivo general y objetivos específicos de acuerdo a la naturaleza del trabajo investigativo. El objetivo general debe responder a la totalidad o globalidad del estudio o propuesta que se pretende realizar, resume el resultado final que se pretende alcanzar con el desarrollo de la propuesta. Los objetivos específicos responden a aspectos singulares, particulares o concretos de la propuesta y se derivan del objetivo general (Varios autores, 2019).

Los objetivos deben estructurarse en función de las preguntas fundamentales que los constituyen: ¿qué?, ¿cómo? y ¿para qué? Los objetivos deben ser planteados utilizando verbos en infinitivo, deben cumplir las principales características de claridad y precisión. Todo objetivo de una investigación debe ser medible, pertinente, relevante, desafiante, bien enfocado y factible de ser alcanzado o realizado. Es preciso recordar que los objetivos deben responder al tema y al problema de la investigación.

\subsection{Justificación}

Esta sección se encuentra constituida por los siguientes componentes: a) presentación breve del tema con la finalidad de atraer la atención del lector; b) la formulación del propósito u objetivo de la propuesta metodológica y tecnológica; c) la explicación y contextualización de la problemática orientadora del trabajo investigativo; d) la formulación del problema que se propone enfrentar; e) la presentación de la idea a defender, la proposición o la hipótesis; f) la explicación de la importancia, la actualidad y la pertinencia de la propuesta metodológica y tecnológica que se propone desarrollar; g) la explicación de las principales directrices del marco metodológico utilizado; h) la descripción del marco conceptual fundamental que respalda a la propuesta; y finalmente, i) una breve descripción de la estructura de la propuesta. En la justificación es necesario utilizar citas bibliográficas, así como la literatura más significativa y actual del tema a escala nacional e internacional (Aguilar-Gordón, 2019b). 


\subsection{Estado de arte}

Esta etapa del proceso consiste en hacer una revisión sistemática del "estado de la cuestión" sobre el tema de la investigación (Carrasco y Calderero, 2009, pág. 31) es el momento en el que el investigador consulta y revisa fuentes de información, busca, recopila y organiza la documentación sobre el tema objeto de la investigación.

El estado del arte se basa en el análisis de las principales publicaciones sobre un tema determinado; su objetivo es definir el estado actual del problema y evaluar las investigaciones realizadas; en términos de Carrasco y Calderero (2009) la revisión documental permite "comprobar si el tema a investigar resulta significativo; permite redefinir el problema o matizarlo, permite situar la investigación dentro de una perspectiva, cuerpo teórico o marco conceptual" (pág. 99).

Su estructura responde a las fases del tema/problema, aportes de investigadores o equipos, cambios en la teoría o las corrientes teóricas principales; problemas sin resolver; tendencias actuales y futuras (Giordanino, 2011). Es una revisión profunda y fundamentada sobre el tema/problema de la investigación. De acuerdo a Delors (1996) en el informe a la UNESCO es lo que se denominaría "estudio recapitulativo".

\subsection{Perspectiva teórica}

Se refiere al cuerpo o desarrollo de la propuesta, implica poner en práctica a lo largo de toda la exposición, una actitud crítica, constructiva y propositiva que deberá tender hacia la interpelación, a efectos de concitar la atención del tema y el problema objeto de estudio. El investigador deberá generar en el lector la capacidad de identificar la intención dialógica de la propuesta y propiciar en él una discusión abierta.

Esta etapa de la propuesta se encuentra conformada por el diálogo del investigador con las teorías, postulados, enfoques, tendencias, autores y corrientes de pensamiento que respalden las afirmaciones realizadas en función del tema y del problema a solucionar.

Esta etapa se encuentra conformada por el conjunto de temas y subtemas que se pretende desarrollar; es una etapa explicativa, interpretativa y propositiva en la que el investigador también genera nuevas teorías, enfoques y perspectivas.

\subsection{Consideraciones metodológicas}

Se refiere al establecimiento de los métodos, técnicas, instrumentos, procedimientos y recursos que se requieren para el desarrollo de la propuesta. La metodología se define por el tema y el problema de la investigación. En el desarrollo de una propuesta se puede optar por metodologías de carácter cualitativo, por metodologías de carácter cuantitativo o metodologías combinadas.

La función de la metodología de una propuesta es asegurar el rigor científico de los resultados, para ello es preciso tener presente los siguientes cuatro criterios básicos establecidos por Lincoln y Guba (1985) citados por Carrasco y Calderero (2009) y que parafraseados a continuación:

a) la veracidad (se manifiesta cuando los datos recogidos responden o se identifican con la realidad; en investigación cuantitativa se refiere a la validez interna y en la metodología cualitativa corresponde con la credibilidad); 
b) la aplicabilidad (posibilidad de aplicar los descubrimientos o resultados obtenidos a otros contextos o situaciones similares; en investigación cuantitativa se denomina validez externa y en metodología cualitativa se convierte en la transferibilidad);

c) la consistencia (señala el grado en que se obtendrán los resultados y si la investigación se replica o se repite; en investigación cuantitativa es lo que se conoce como fiabilidad y en investigación cualitativa se denomina como dependencia); $y$,

d) la neutralidad (significa la seguridad de que los datos no están sesgados, en investigación cuantitativa equivale a lo que se denomina objetividad y en investigación cualitativa se identifica con lo que se conoce como confirmación).

\subsubsection{Métodos}

Son un conjunto de procedimientos organizados sistemáticamente que nos conducen a la consecución de los objetivos planteados en la investigación. Entre los métodos que pueden ser de utilidad se encuentran los métodos lógicos científicos: inductivo, deductivo y combinado de acuerdo al tema y problema de la investigación.

Si la propuesta se dirige a resolver un problema concreto de contextos y sujetos determinados, singulares o particulares, generalmente, utiliza el método científico orientado por las siguientes etapas: observación de un fenómeno o realidad problemática concreta; formulación de hipótesis, proposición o idea a defender; experimentación; análisis, comparación, generación de nueva teoría, conclusión o formulación de una nueva ley.

Al contrario, si la propuesta se dirige a la solución de una necesidad teórica identificada a nivel de los contenidos del corpus científico utilizará la deducción como punto de partida para demostrar su validez en la práctica concreta, para ello se propone el siguiente proceso: el marco teórico referente o previo entendido como el corpus de ideas o teorías que toma el investigador como guía de su trabajo; el planteamiento del problema (teórico o práctico) para el que buscará una respuesta; la formulación de una hipótesis, proposición o idea a defender; la deducción de las consecuencias observadas; la corroboración o refutación de hipótesis mediante el uso del razonamiento (de carácter deductivo) con miras a aniquilar y reemplazar una teoría; parafraseando a Finol y Nava (1993) las consecuencias posteriores determinadas por los resultados obtenidos, por el impacto social y cultural del nuevo descubrimiento.

Es importante considerar que la propuesta puede auxiliarse de métodos filosóficos como el fenomenológico; el hermenéutico; el estudio de casos; el etnográfico; etc.

\subsubsection{Método fenomenológico}

Carrasco y Calderero (2009) sostienen que el método fenomenológico "es la investigación sistemática de la subjetividad" (pág. 108). Además, en términos de Bullington y Karlson (1984) "su meta es el estudio del mundo tal como se nos presenta en y a través de la conciencia” (pág. 51).

Por su parte, Patton (1999) citado por Carrasco y Calderero (2009) establece que el método fenomenológico se centra en "cómo las personas comprenden los significados de lo que sucede" (pág. 108) en la medida en que los investigadores estudian las formas cualitativamente diferentes con las que los sujetos experimentan o piensan sobre varios fenómenos. El método fenomenológico se auxilia del método hermenéutico o de la interpretación cuyo propósito es descubrir las formas de comprensión que las personas tienen sobre los fenómenos específicos para encuadrarlos dentro de categorías conceptuales, en tal sentido, realizan clasificaciones sobre las concepciones que los sujetos

Licencia Creative Commons Atribución 4.0 Internacional (CC BY 4.0)

Revista Cátedra, 2 (2), pp. 94-110, mayo-agosto 2019. e-ISSN: 2631-2875

https://doi.org/10.29166/catedra.v2i2.1708 
tienen acerca del mundo y de la realidad con miras a que los resultados obtenidos puedan ser generalizables y puedan ser aplicables a diversas situaciones y contextos.

Esta metodología fenomenológica-hermenéutica resulta apropiada para investigar una multitud de cuestiones educativas, por ejemplo: interpretaciones que realizan los estudiantes acerca de la manera de proceder de los docentes en el aula; percepciones de los estudiantes sobre las formas de enseñanza; etc.

\subsubsection{Método del estudio de casos}

Este método implica el análisis de una situación real en la que existen necesidades y problemas que requieren oportunas respuestas y soluciones; consiste en una descripción y análisis detallados de unidades sociales o entidades educativas específicas. A decir de Carrasco y Calderero (2009) este método "se sitúa dentro del enfoque "ideográfico", ya que pretende la comprensión profunda de la realidad singular-individuo, familia, grupo, institución social, comunidad, etc." (pág. 110).

Este tipo de método resulta apropiado para el estudio de una situación concreta en corto tiempo, puede hacerse en equipos de trabajo dentro de un marco de discusión y de debate en el que el intercambio de ideas permite la comprensión adecuada del problema o fenómeno investigado, enriquece la propuesta y arroja interesantes resultados.

\subsubsection{Método etnográfico}

Este método se propone descubrir creencias, valores, interacciones, comportamientos, perspectivas, conductas, motivaciones de las personas o de un grupo social-educativo determinando y el modo en que todo esto se desarrolla con el tiempo; lo que cuenta para este método es el significado y las interpretaciones. La etnografía educativa "estudia las distintas áreas de la vida social de la escuela" (Carrasco y Calderero, 2009, pág. 112); de modo que describe, explica e interpreta los fenómenos educativos que tienen lugar en el contexto de la institución educativa. Siguiendo el estudio de Goetz, J. y LeCompte, M. (1988), las fases que conforman el proceso de investigación etnográfica son cuatro:

a) Cuestiones relativas a la investigación y marcos teóricos preliminares.

b) Acceso del investigador al escenario, selección de los informantes clave, inicio de las entrevistas y selección de las estrategias de obtención de información y de registro. c) Recogida de información. d) Análisis e interpretación de la información (pág.172).

Siempre la investigación se inicia con una idea global o temática del trabajo, se intenta comprender un sistema con los significados propios de los participantes. La etnografía es un medio eficaz para el análisis crítico y reflexivo de las acciones y prácticas docentes.

Así mismo, Wilcox (1993) sostiene que las aplicaciones de la etnografía en la educación se resumen en dos grandes líneas maestras: “a) Exploración de la escuela como instrumento de transmisión cultural en el aula, comunicando un conjunto complejo de actitudes, normas de conducta valores y expectativas.... y, b) Exploración del conflicto cultural en el aula" (pág. 103). En el primer caso se incluyen investigaciones sobre el currículo oculto o sobre el aula como agente de socialización. En el segundo caso se pueden considerar las investigaciones acerca del enfrentamiento entre cultura institucional y organización familiar y sus repercusiones en el aprendizaje, estilos de aprendizaje, formas de pensamiento, normas de interacción, etc.

Licencia Creative Commons Atribución 4.0 Internacional (CC BY 4.0)

Revista Cátedra, 2 (2), pp. 94-110, mayo-agosto 2019. e-ISSN: 2631-2875

https://doi.org/10.29166/catedra.v2i2.1708 


\subsubsection{Técnicas}

En términos generales, las técnicas son entendidas como procedimientos metodológicos y sistemáticos que se encargan de operativizar e implementar los métodos de investigación y que permiten la recopilación eficaz e inmediata de la información. Las técnicas de utilidad para el desarrollo de una propuesta son varias y son empleadas de acuerdo al tema, al problema investigado, al contexto, a la circunstancia, al tiempo e inclusive de acuerdo a la capacidad del investigador; entre ellas tenemos: la técnica bibliográfica o documental; la observación; la entrevista; la encuesta; el fichaje; el muestreo; el test; el grupo focal. Todas ellas en su variedad de enfoques contribuyen para la recolección de la información necesaria en una investigación.

\subsubsection{Instrumentos}

Los instrumentos de investigación son las herramientas empleadas por el investigador para la recopilación de la información conforme al tema, al problema y al objetivo de la investigación.

Hernández (1991) sostiene que "el instrumento de medición adecuado es el que registra los datos observables, de forma que representen verdaderamente a las variables que el investigador tiene por objeto" (pág. 123), además este autor manifiesta que a pesar de existir diferentes tipos de instrumentos de investigación, "existe un procedimiento general que se puede adaptar a cada uno de los tipos de instrumentos" (Hernández, 1991, pág. 124); este procedimiento para construir instrumentos de investigación consta de los siguientes pasos: "listar las variables que se van a medir; revisar la definición conceptual de las variables y comprender su significado; revisar cómo han sido definidas operacionalmente las variables; elegir el instrumento y adaptarlo al contexto de la investigación" (Hernández, 1991, pág. 125).

En general, los instrumentos utilizados son diseñados de acuerdo a la técnica seleccionada, pues cada técnica conlleva el uso de su propio instrumento tal como se explica en el Cuadro 1.

\begin{tabular}{cl}
\hline $\begin{array}{c}\text { Técnica de } \\
\text { Investigación }\end{array}$ & Instrumento \\
\hline Observación & $\begin{array}{l}\text { Guía de observación } \\
\text { Lista de cotejo }\end{array}$ \\
Fichaje & Tarjetas \\
Encuesta & Cuestionario \\
Entrevista & Guía de entrevista \\
Grupo focal & Pauta de discusión \\
Test & Guía de discusión \\
\hline
\end{tabular}

Cuadro 1. Técnicas de instrumentos de investigación

\subsubsection{Procedimientos}

Consiste en la descripción de los pasos, tácticas, estrategias y acciones que ser proponen para llevar a cabo la investigación que consolidará la propuesta metodológica y tecnológica. Entre estos procedimientos se pueden mencionar:

1. Identificación del tema o problema de la propuesta;

2. Revisión documental relevante relacionada con el tema, el problema y las cuestiones planteadas con el objeto de conocer el estado de la cuestión; 
3. Acciones relacionadas con la realización del plan o del diseño de la propuesta (selección del lugar o del escenario en donde se va a desarrollar la propuesta; las acciones relacionadas con el acceso al lugar, la receptividad, la factibilidad, las características, predisposición de los participantes; la selección del método; el tiempo que durará el estudio, los imprevistos, los recursos con los que se cuenta para el desarrollo de la investigación);

4. Actividades vinculadas al inicio del estudio (conocer el contexto, las normas, la selección de participantes o el muestreo para el desarrollo de la propuesta);

5. Tareas sobre la recogida y análisis de los datos (la selección de las técnicas, instrumentos y procedimientos; el análisis de la información, el aseguramiento del rigor del trabajo investigativo);

6. Acciones y estrategias en relación a la terminación del estudio;

7. Las tareas en función de la elaboración del informe de la investigación y la redacción de la propuesta.

\subsubsection{Recursos}

Constituyen los insumos que se requieren para llevar adelante una propuesta metodológica y tecnológica, éstos son de distintas clases: humanos, materiales, económicos, financieros, técnicos y tecnológicos. Todos ellos son activos esenciales y actúan como en un sistema que permite la ejecución de un programa, plan de acción, proyecto o propuesta. La existencia de todos los recursos de investigación antes mencionados hace posible el cumplimiento de los objetivos establecidos, conlleva e integra la actividad científica y coadyuvan a la resolución del problema de la investigación. Existe una clara conexión entre recursos de investigación y método científico, pues a pesar de ser realidades diferentes son complementarias y se requieren mutuamente para la resolución de un problema, para la obtención y para el procesamiento, organización y sistematización de la información y en fin para todo el proceso investigativo. Solamente es factible obtener información del sistema investigado gracias a la existencia de los recursos principalmente el referido al talento humano.

\section{Resultados}

Expone de manera objetiva los principales hallazgos; ofrece una visión de las implicaciones del trabajo, las limitaciones, la respuesta tentativa al problema, las relaciones con el objetivo de la investigación y las posibles líneas de continuidad (Aguilar-Gordón, 2019b). Los resultados deberán ser reales en función de los datos obtenidos, analizados, procesados y sistematizados en la investigación.

\section{Conclusiones}

Se desprenden del trabajo investigativo, están asociadas a la totalidad del proceso, pueden estar coligadas con las recomendaciones, evaluaciones, aplicaciones, sugerencias, nuevas relaciones e hipótesis aceptadas o rechazadas, el material y los métodos empleados.

\subsection{Condiciones de las conclusiones}

- Deben encontrarse debidamente justificadas

- Deben responder a los objetivos y al problema de la investigación

- Deben incluir las deducciones, inferencias y nuevas líneas para futuras investigaciones

- Deben ser redactadas de manera clara y precisa

- No deben hacer juicios de valor

- No deben repetir o duplicar los datos 
- No agregan nueva información

- Deben aparecer en una secuencia lógica y coherente al final del texto.

\section{Cronograma}

Es la planificación y organización temporal de las fases, actividades y tareas previstas para el desarrollo de la propuesta, se utiliza el gráfico de Gantt ideado por Henry Laurence Gantt. El gráfico de Gantt es "una herramienta visual para la planificación y programación de las actividades o tareas sobre una línea de tiempo" (Riviere y Riviere, 2017, pág. 8). Esta herramienta permite al usuario establecer gráficamente la duración, el comienzo de cada una de las actividades, el seguimiento y el control de la totalidad de la planificación. El cronograma encadena y sincroniza tiempo y acciones para el cumplimiento de lo programado en la investigación; refleja dificultades y aciertos del proceso investigativo.

\section{Bibliografía}

"Es el conjunto de obras utilizadas en la estructuración" (Aguilar-Gordón, 2019b, pág. 360) de la propuesta metodológica. La bibliografía deberá estar presentada en orden alfabético considerando el primer apellido del autor y en el formato sugerido en las normas internacionales APA en su sexta edición. Deben incluirse única y exclusivamente las obras y los autores realmente utilizados en la investigación y "su número debe ser suficiente para contextualizar el marco teórico con criterios de actualidad e importancia” (Aguilar-Gordón, 2019b, págs. 358-359).

\section{Conclusiones}

La propuesta metodológica es uno de los mecanismos para lograr una verdadera integración de saberes, contribuye para la formación integral de los seres humanos; constituye una opción para obtener una titulación a nivel de grado y de postgrado.

Armoniza la teoría con la práctica; es de carácter humanista-científica-constructivista, tiende a la transformación social. Este trabajo plantea un conjunto de pautas para la estructuración de una propuesta metodológica crítica, creativa e innovadora.

Se propone responder a la realidad del contexto, a las necesidades concretas de una comunidad determinada y a unos sujetos complejos en una sociedad compleja. La propuesta metodológica se convierte en una unidad en la que coexisten contenidos, conceptualizaciones, argumentaciones, experiencias concretas, investigación y práctica real, se orienta hacia la solución de problemas, a la transformación y al perfeccionamiento humano.

La propuesta metodológica es clara y precisa, responde a las preguntas fundamentales: ¿qué? ¿en dónde? ¿cómo? ¿cuándo? ¿con qué?; describe escenarios, problemas y establece alternativas de solución. 


\section{Bibliografía}

Aguilar-Gordón, F. (2010). Percepción y meta-cognición en la educación: una mirada desde América Latina, en Revista Sophia: Colección de Filosofía de la Educación, N. 8, pp. 147-195, DOI: https://doi.org/10.17163/soph.n8.2010.06, Quito-Ecuador, recuperado de: https://sophia.ups.edu.ec/index.php/sophia/article/view/8.2010.06

Aguilar-Gordón, F. (2019a). Fundamento, evolución, nodos críticos y desafíos de la educación ecuatoriana actual, en Revista Actualidades Investigativas en Educación, Volumen 19, Número 1, pp. 1-31, DOI: 10.15517/aie.v19i1.35715, Costa Rica, recuperado de: https://revistas.ucr.ac.cr/index.php/aie/article/view/35715

Aguilar-Gordón, F. (2019b). Normas Editoriales, en Sophia: Colección de Filosofía de la Educación, N. 26. Universidad Politécnica Salesiana, Abya-Yala, Quito, recuperado de: file://C:/Users/Floralba/Downloads/157-116-PB.pdf

Bullington, J. y Karlson, G. (1984). Introducción al método fenomenológico de la investigación, en Revista Escandinava de Psicología, N. 25, pp. 51-63

Briones, G. (2013). Metodología y técnicas de Investigación para las Ciencias Sociales. Trillas. Cuarta reimpresión. México D.F. México.

Consejo de Educación Superior (CES) (2017). Reglamento de Régimen Académico, en Gaceta oficial, recuperado de: http://webcache.googleusercontent.com/search?q=cache:heo2TY phLEJ:www.ces.g ob.ec/doc/Reglamentos/2017/Abril/reglamento\%2520de\%2520regimen\%2520ac ademico $\% 2520$ codificacion.pdf $+\& c d=1 \& h l=e s \& c t=c \ln \& g \mathrm{gl}=e c \& c l i e n t=$ firefox-b-d

Delors, J. (1996) La educación encierra un tesoro. Informe a la UNESCO de la Comisión Internacional sobre la Educación para el Siglo XXI. Santillana, Ediciones UNESCO, Madrid-España, recuperado de: www.unesco.org/education/pdf/DELORS S.PDF

Finol, T. y Nava, H. (1993). Procesos y productos en la Investigación Documental. Ediluz. Universidad del Zulia. Maracaibo.

Hernández, R. (1991). Metodología de la Investigación. Mc - Graw- Hill. México D.F. México.

Carrasco, J. y Calderero, J. (2009). Aprendo a Investigar en Educación. Ediciones RIALP, S.A., Madrid.

Goetz, J. y LeCompte, M. (1988). Etnografía y diseño cualitativo en investigación educativa. Madrid: Morata.

Giordanino, E. (2011). Las revistas científicas: estructura y normalización. Pautas y evaluación, en Acuerdo de Bibliotecas Universitarias de Córdoba (ABUC), Córdoba, recuperado de: https://www.aacademica.org/egiordanino/29.pdf

Lara, R. (1998). Tecnología: Conceptos, problema y perspectivas. Madrid, España: Editorial Siglo XXXI. 
Marchetto, M. (2006). Modelo teórico interpretativo del proceso de investigación desde un enfoque innovador en el IUETAEB. Tesis doctoral. Caracas, Venezuela: USM.

Pérez Juste, R. y otros (1981). Psicología Experimental I. Vol. I, Madrid: UNED.

Reviere, C. y Reviere, O. (2017). ¿Qué es un Diagrama de Gantt? Ed. Calleja, Madrid-España

Torres, J. (2015). ¿Qué es la propuesta metodológica IDEA?, en Iberoamérica Divulga. IBERCIENCIA. Comunidad de Educadores para la Cultura Científica. Universidad Autónoma Chapingo, Texcoco. México, recuperado de: https://www.oei.es/historico/divulgacioncientifica/

Varios Autores (2019). Significado. Diccionario online, recuperado de https://www.significados.com/objetivo-de-investigacion/

Wilcox, K. (1993). La etnografía como una metodología y sus aplicaciones al estudio de la escuela, en Lecturas de Antropología para educadores. Madrid: Trotta. 


\section{Autora}

FLORALBA AGUILAR-GORDóN obtuvo su título de Doctora en Filosofía por la Pontificia Universidad Católica del Ecuador, en 1996. Obtuvo su título de Magíster en Educación, mención Educación Superior por la Universidad Tecnológica América, en 2008. Obtuvo su título de Magíster en Tecnología aplicada la educación por la Universidad de Alicante, la Universidad Autónoma de Barcelona y la Universidad Carlos III de Madrid, en 2010. Obtuvo su título de Magíster en Educación a Distancia por la Universidad Técnica Particular de Loja, en 2010. Obtuvo su título de Experto en Analítica del conocimiento por la Universidad Internacional de la Rioja de España, en 2016. Obtuvo su título de Especialista en Planificación Curricular y Organización de Sistemas de Educación a Distancia por la Universidad Técnica Particular de Loja. Obtuvo su Diplomado Superior en Currículo y Didáctica por la Universidad Tecnológica América. Obtuvo su Diplomado Superior en Transformación Educativa por la Universidad Multiversidad Edgar Morin de México en 2009. Obtuvo su Diplomado Superior en e-learning, por el Instituto Tecnológico de Madrid, en 2016. Obtuvo su Diplomado Superior en Investigación Educativa por la Universidad Tecnológica América. Obtuvo su Diplomado Superior en Fundamentos de la Educación a Distancia e Investigación por la Universidad Técnica Particular de Loja. Obtuvo su Diploma Superior en Aprendizaje cooperativo por la Universidad Católica de Brasilia, en 2002. Obtuvo su certificación de tutora internacional acreditada por la Universidad Católica de Brasilia en 2004. Obtuvo su Diploma Superior en Tecnología, Gerencia y Liderazgo por la Universidad Tecnológica América. Obtuvo su título de Licenciada en Filosofía por la Facultad de Ciencias Humanas de la Pontificia Universidad Católica del Ecuador, en 1991. Obtuvo su título de Licenciada en Ciencias Sociales, Políticas y Económicas por la Escuela de Ciencias Jurídicas de la Universidad Técnica Particular de Loja, en 2007. Obtuvo el título de Abogada por la Escuela de Ciencias Jurídicas de la Universidad Técnica Particular de Loja, en 2013.

Actualmente es profesora titular de la Universidad Politécnica Salesiana. Es Editora Jefa de la Revista Sophia: Colección de Filosofía de la Educación editada por la Universidad Politécnica Salesiana del Ecuador indexada en más de 60 índices y bases de datos de reconocido prestigio internacional. Es Coordinadora del Grupo de Investigación en Filosofía de la Educación (GIFE). Miembro del Consejo Científico y revisora internacional de importantes revistas de Ecuador, España, Colombia, Uruguay, Chile, México y Costa Rica. 